\title{
Almost Secure (1-Round, $n$-Channel) Message Transmission Scheme
}

\author{
Kaoru Kurosawa ${ }^{1}$ and Kazuhiro Suzuki ${ }^{2}$ \\ 1 Department of Computer and Information Sciences, Ibaraki University, Japan \\ kurosawa@mx.ibaraki.ac.jp \\ 2 Venture Business Laboratory, Ibaraki University, Japan \\ tutetuti@dream.com
}

\begin{abstract}
It is known that perfectly secure (1-round, $n$-channel) message transmission (MT) schemes exist if and only if $n \geq 3 t+1$, where $t$ is the number of channels that the adversary can corrupt. Then does there exist an almost secure MT scheme for $n=2 t+1$ ? In this paper, we first sum up a number flaws of the previous almost secure MT scheme presented at Crypto $2004^{3}$. We next show an equivalence between almost secure MT schemes and secret sharing schemes with cheaters. By using our equivalence, we derive a lower bound on the communication complexity of almost secure MT schemes. Finally, we present a near optimum scheme which meets our bound approximately. This is the first construction of provably secure almost secure (1-round, $n$-channel) MT schemes for $n=2 t+1$.
\end{abstract}

Keywords: Private and reliable transmission, information theoretic security, communication efficiency

\section{Introduction}

\subsection{Message Transmission Scheme}

The model of ( $r$-round, $n$-channel) message transmission schemes was introduced by Dolev et al. [2]. In this model, there are $n$ channels between a sender and a receiver while they share no keys. The sender wishes to send a secret $s$ to the receiver in $r$-rounds securely and reliably. An adversary $\mathbf{A}$ can observe and forge the messages sent through $t$ out of $n$ channels.

We say that a ( $r$-round, $n$-channel) message transmission scheme is perfectly $t$-secure if $\mathbf{A}$ learns no information on $s$ (perfect privacy), and the receiver can output $\hat{s}=s$ correctly (perfect reliability) for any infinitely powerful adversary A who can corrupt at most $t$ channels (in information theoretic sense). ${ }^{4}$ Dolev et al. showed that [2]

\footnotetext{
3 The authors already noted in thier presentation at Crypto'2004 that their scheme was flawed. It was Ronald Cramer who informed the authors of the flaw.

${ }^{4}$ Dolev et al. called it a perfectly secure message transmission scheme [2].
} 
$-n \geq 3 t+1$ is necessary and sufficient for $r=1$, and

$-n \geq 2 t+1$ is necessary and sufficient for $r=2$

to achieve perfect $t$-security.

A perfectly $t$-secure scheme with optimum communication complexity is known for $r=1$ and $n=3 t+1[2,6]$. Based on the work of [5,6], Agarwal et al. showed an asymptotically optimum perfectly $t$-secure scheme for $r=2$ and $n=2 t+1[1]$.

\subsection{Secret Sharing Scheme with Cheaters}

Tompa and Woll introduced a problem of cheating in $(k, n)$ threshold secret sharing schemes [7]. In this problem $k-1$ malicious participants aim to cheat an honest one by opening forged shares and causing the honest participant to reconstruct the wrong secret.

Ogata et al. derived a tight lower bound on the size of shares $\left|\mathcal{V}_{i}\right|$ for secret sharing schemes that protects against this type of attack: $\left|\mathcal{V}_{i}\right| \geq(|\mathcal{S}|-1) / \delta+1$, where $\mathcal{V}_{i}$ denotes the set of shares of participant $P_{i}, \mathcal{S}$ denotes the set of secrets, and $\delta$ denotes the cheating probability [4].

They also presented an optimum scheme, which meets the equality of their bound by using "difference sets" [4].

\subsection{Our Contribution}

As we mentioned, it is known that perfectly secure (1-round, $n$-channel) message transmission schemes exist if and only if $n \geq 3 t+1$, where $t$ is the number of channels that adversary can corrupt. Then does there exist an almost secure scheme for $n=2 t+1$ ? At Crypto 2004, Srinathan et al. [6, Sec.5] proposed an almost secure (1-round, $n$-channel) message transmission scheme for $n=2 t+1$. However, the authors already noted in thier presentation at Crypto'2004 that their scheme was flawed.

In this paper, we first sum up a number of flaws of the above scheme. (Actually, they showed two schemes in [6], a perfectly $t$-secure scheme and an almost secure scheme. Agarwal et al. showed a flaw of the former one [1].)

Table 1. Previous Work and Our Contribution

\begin{tabular}{|l|c|c|}
\hline & Perfectly $t$-secure & Almost secure \\
\hline \hline$r=1$ & $n \geq 3 t+1$ & $\begin{array}{c}n=2 t+1 \\
\text { This paper }\end{array}$ \\
\hline$r=2$ & $n \geq 2 t+1$ & - \\
\hline
\end{tabular}

\footnotetext{
$\overline{{ }^{5}|\mathcal{X}| \text { denotes }}$ the cardinality of a set $\mathcal{X}$.
} 
We next show an equivalence between almost secure (1-round, $n$-channel) message transmission schemes with $n=2 t+1$ and secret sharing schemes with cheaters. By using our equivalence, we derive a lower bound on the communication complexity of almost secure (1-round, $n$-channel) message transmission schemes (in the above sense) such that

$$
\left|\mathcal{X}_{i}\right| \geq(|\mathcal{S}|-1) / \delta+1
$$

where $\mathcal{X}_{i}$ denotes the set of messages sent through the $i$ th channel and $\mathcal{S}$ denotes the set of secrets which the sender wishes to send to the receiver.

We finally show a near optimum scheme which meets our bound approximately. This is the first construction of almost secure (1-round, $n$-channel) message transmission schemes for $n=2 t+1$.

Our results imply that $n \geq 2 t+1$ is necessary and sufficient for almost secure (1-round, $n$-channel) message transmission schemes.

\section{Flaw of the Previous Almost Secure MT Scheme}

In this section, we sum up a number of flaws of the previous almost secure (1round, $n$-channel) message transmission scheme [6, Sec.5]. ${ }^{6}$ Let $n=2 t+1$ in what follows.

\subsection{Previous Almost Secure Message Transmission Scheme}

Their scheme [6, Sec.5] is described is as follows. For simplicity, let $\mathbb{F}$ be a finite field $G F(q)$ such that $q$ is a prime, and assume that the sender wishes to send a secret $s=\left(s_{1}, \ldots, s_{t+1}\right)$ to the receiver, where each $s_{i}$ is an element of $\mathbb{F} .{ }^{7}$

- Enc. The sender computes a ciphertext $\left(x_{1}, \cdots, x_{n}\right)$ from $s=\left(s_{1}, \ldots, s_{t+1}\right)$ as follows.

1. Randomly select $n$ polynomials $p_{1}(x), \cdots, p_{n}(x)$ of degree at most $t$ over $\mathbb{F}$ such that

$$
Q(1)=s_{1}, \cdots, Q(t+1)=s_{t+1},
$$

where ${ }^{8} Q(x)=p_{1}(0)+p_{2}(0) x+p_{3}(0) x^{2}+\cdots+p_{n}(0) x^{n-1}$.

2. For each $(i, j)$ with $i \neq j$, randomly select one of the $t$ points of intersection of $p_{i}$ and $p_{j}$ so that $r_{i j} \neq r_{j i}$ (denote the selected point by $\left.r_{i j}\right)$.

3. For each $i$, let $x_{i}=\left(p_{i}(x), r_{i j}\right.$ for all $\left.j \neq i\right)$.

4. Output $\left(x_{1}, x_{2}, \ldots, x_{n}\right)$.

- Dec. The receiver computes $s=\left(s_{1}, \ldots, s_{t+1}\right)$ or $\perp$ from $\left(\hat{x}_{1}, \hat{x}_{2}, \ldots, \hat{x}_{n}\right)$ as follows, where $\hat{x}_{i}=\left(\hat{p}_{i}(x), \hat{r}_{i j}\right.$ for all $\left.j \neq i\right)$.

\footnotetext{
6 They called it a Las Vegas scheme.

${ }^{7}$ In $\left[6\right.$, Sec.5], the sender sends a message $m=\left(m_{1}, \cdots, m_{t+1}\right)$ to the receiver by broadcasting $y=m+s$ through all the channels.

${ }^{8}$ In $\left[6\right.$, Sec.5], they wrote this as $s=\operatorname{EXTRAND}\left(p_{1}(0), \cdots, p_{n}(0)\right)$.
} 
1. Set $\Lambda=\{1,2, \ldots, n\}$.

2. We say that the $i$-th channel $c_{i}$ contradicts the $j$-th channel $c h_{j}$ if $\hat{p}_{i}$ and $\hat{p}_{j}$ do not intersect at $\hat{r}_{i j}$.

3. For each $i$, if $c h_{i}$ is contradicted by at least $t+1$ channels then remove $i$ from $\Lambda$.

4. If $c h_{i}$ contradicts $c h_{j}$ for some $i, j \in \Lambda$ then output failure.

5. If $|\Lambda| \leq t$, then output failure.

6. At this point, $\hat{p}_{i}=p_{i}$ for all $i \in \Lambda$ and $|\Lambda| \geq t+1$.

Derive all the polynomials $p_{1}, \ldots, p_{n}$ from $\hat{p}_{i}$ and $\hat{r}_{i j}(i \in \Lambda)$.

7. Compute $s$ as $s=[Q(1), \ldots, Q(t+1)]$.

Srinathan et al. claimed the following lemmas for adversaries who can corrupts at most $t$ out of $n$ channels [6, Sec.5].

Lemma 1. [6, Lemma 11] Reliability. The receiver will never output an incorrect value.

Lemma 2. [6, Lemma 13] Perfect Privacy. The adversary gains no information about the secret.

\section{$2.2 \quad$ Flaws}

We show that the above two lemmas do not hold. In the above scheme, it is important to choose $p_{1}, \cdots, p_{n}$ randomly because otherwise we cannot ensure the perfect privacy. However, if the sender chooses $p_{1}, \cdots, p_{n}$ randomly, it has the following problems. For simplicity, suppose that $t=2$ and $n=2 t+1=5$. (It is easy to generalize the following argument to any $t \geq 2$.)

- Sender's problem: Since the polynomials $p_{1}, \ldots, p_{5}$ are randomly chosen, it can happen that some $p_{i}$ and $p_{j}$ do not intersect or intersect at one point. In these cases, the sender cannot execute Step 2 of Enc.

- Perfect Privacy: Suppose that the adversary A corrupts $t=2$ channels 1 and 2. In most cases, $\mathbf{A}$ has no information on $s_{1}, s_{2}, s_{3}$ because eq. (1) has $t+1=3$ equations and 3 unknown variables $p_{3}(0), p_{4}(0)$ and $p_{5}(0)$, where $p_{3}(0), p_{4}(0)$ and $p_{5}(0)$ are randomly chosen.

However, with nonzero probability, it happens that $p_{1}(x)$ and $p_{3}(x)$ intersect at $x=0$ and hence $r_{1,3}=0$. In this case, $\mathbf{A}$ can compute $p_{3}(0)$, and she knows 3 values, $p_{1}(0), p_{2}(0)$ and $p_{3}(0)$. Consequently, A has only 2 unknown variables $p_{4}(0)$ and $p_{5}(0)$ in eq.(1). This means that $\mathbf{A}$ can learn some information on $s=\left(s_{1}, s_{2}, s_{3}\right)$ with nonzero probability. Therefore Lemma 2 (perfect privacy) does not hold.

- Reliability: Since the polynomials $p_{1}(x), \ldots, p_{5}(x)$ are all randomly chosen, it can happen that

$$
\begin{aligned}
& b_{1}=p_{1}\left(a_{1}\right)=\cdots=p_{5}\left(a_{1}\right) \\
& b_{2}=p_{1}\left(a_{2}\right)=\cdots=p_{5}\left(a_{2}\right)
\end{aligned}
$$


with nonzero probability. That is, all polynomials go through $\left(a_{1}, b_{1}\right)$ and $\left(a_{2}, b_{2}\right)$. In this case, the sender will set $r_{i j}=a_{1}$ and $r_{j i}=a_{2}$ for each pair $i<j$.

Now consider an adversary $\mathbf{A}$ who corrupts channel 1 and replaces $p_{1}(x)$ with a random polynomial $p_{1}^{\prime}(x)$. Then it can still happen that $p_{1}^{\prime}$ passes through $\left(a_{1}, b_{1}\right)$ and $\left(a_{2}, b_{2}\right)$ with nonzero probability. In this case, the receiver accepts $p_{1}^{\prime}$. Hence the receiver outputs $\hat{s} \neq s$ because $p_{1}^{\prime}(0) \neq p_{1}(0)$. After all, the receiver outputs $\hat{s} \neq s$ with nonzero probability. Therefore, Lemma 1 does not hold.

We cannot fix these flaws. To correct these flaws, Enc must choose $p_{1}, \cdots, p_{5}$ in such a way that

$-p_{i}$ and $p_{j}$ intersect at at least two points,

$-r_{i j} \neq 0$,

- and all intersection points are distinct

for each pair of $(i, j)$. However, if so, the perfect privacy does not hold because $p_{1}, \cdots, p_{5}$ are not random.

Suppose that the adversary A corrupts $t=2$ channels 1 and 2 . Then she learns the values of $p_{1}(0), p_{2}(0)$. Hence she knows that $p_{3}(0), \ldots, p_{5}(0)$ are not elements of $\left\{p_{1}(0), p_{2}(0)\right\}$. That is, $p_{3}(0), \ldots, p_{5}(0)$ are not randomly chosen from $\mathbb{F}$. Hence she can learn some information on $s$ from eq.(1).

\section{Model}

In this section, we define a model of Almost Secure (1-round, $n$-channel) message transmission schemes formally. In the model, there are $n$ channels between a sender and a receiver. The sender wishes to send a secret $s$ to the receiver secretly and reliably in one-round without sharing any keys. An adversary can observe and forge the messages sent through at most $t$ out of $n$ channels.

A (1-round, $n$-channel) message transmission scheme consists of a pair of algorithms (Enc, Dec) as follows. Let $\mathcal{S}$ denote the set of secrets.

- Enc is a probabilistic encryption algorithm which takes a secret $s \in \mathcal{S}$ as an input, and outputs a ciphertext $\left(x_{1}, \cdots, x_{n}\right)$, where $x_{i}$ is the $i$-th channel's message.

- Dec is a deterministic decryption algorithm which takes an alleged ciphertext $\left(\hat{x}_{1}, \cdots, \hat{x}_{n}\right)$ and outputs $\hat{s} \in \mathcal{S}$ or failure.

We require that $\operatorname{Dec}(\operatorname{Enc}(s))=s$ for any $s \in \mathcal{S}$. We assume a certain probability distribution over $\mathcal{S}$, and let $S$ denote the random variable. Let $X_{i}$ denote the random variable induced by $x_{i}$, and $\mathcal{X}_{i}$ denote the possible set of $x_{i}$ for $1 \leq i \leq n$.

To define the security, we consider the following game among the sender, the receiver and an adversary $\mathbf{A}$, where $\mathbf{A}$ is a (infinitely powerful) probabilistic Turing machine. 
1. A chooses $t$ channels, $i_{1}, \cdots, i_{t}$.

2. The sender chooses $s \in \mathcal{S}$ according to the distribution over $\mathcal{S}$, and uses Enc to compute $x_{1}, \cdots, x_{n}$. Then $x_{i}$ is sent to the receiver through channel $i$ for $1 \leq i \leq n$.

3. A observes $x_{i_{1}}, \cdots, x_{i_{t}}$, and forges them to $x_{i_{1}}^{\prime}, \cdots, x_{i_{t}}^{\prime}$. We allow $x_{i_{j}}^{\prime}$ to be the null string for $1 \leq j \leq t$.

4. The receiver receives $\hat{x}_{i}$ through channel $i$ for $1 \leq i \leq n$, and uses Dec to compute

$$
\operatorname{Dec}\left(\hat{x}_{1}, \cdots, \hat{x}_{n}\right)=\hat{s} \text { or failure. }
$$

Definition 1. We say that a (1-round, $n$-channel) message transmission scheme is $(t, \delta)$-secure if the following conditions are satisfied for any adversary $\mathbf{A}$ who can corrupt at most $t$ out of $n$ channels.

Privacy. A learns no information on s. More precisely,

$$
\operatorname{Pr}\left(S=s \mid X_{i_{1}}=x_{i_{1}}, \cdots, X_{i_{t}}=x_{i_{t}}\right)=\operatorname{Pr}(S=s)
$$

for any $s \in \mathcal{S}$ and any possible $x_{i_{1}}, \cdots, x_{i_{t}}$.

General Reliability. The receiver outputs $\hat{s}=s$ or failure. (He never outputs a wrong secret.)

Trivial Reliability. If the $t$ forged messages $x_{i_{1}}^{\prime}, \cdots, x_{i_{t}}^{\prime}$ are all null strings, then Dec outputs $\hat{s}=s$.

Failure.

$$
\operatorname{Pr}(\text { Dec outputs failure })<\delta \text {. }
$$

(The trivial reliability means that if $t$ channel fail to deliver messages, then Dec outputs $\hat{s}=s$. Hence this is a reasonable requirement.)

\section{Secret Sharing Scheme with Cheaters}

In the model of secret sharing schemes, there is a probabilistic Turing machine $D$ called a dealer. $S$ denotes a random variable distributed over a finite set $\mathcal{S}$, and $s \in \mathcal{S}$ is called a secret. On input $s \in \mathcal{S}, D$ outputs $\left(v_{1}, \ldots, v_{n}\right)$ according to some fixed probability distribution. For $1 \leq i \leq n$, each participant $P_{i}$ holds $v_{i}$ as his share. $V_{i}$ denotes the random variable induced by $v_{i}$. Let $\mathcal{V}_{i}=\left\{v_{i} \mid\right.$ $\left.\operatorname{Pr}\left[V_{i}=v_{i}\right]>0\right\} . \mathcal{V}_{i}$ is the set of possible shares held by $P_{i}$.

Definition 2. We say that $D$ is a $(k, n)$ threshold secret sharing scheme for $S$ if the following two requirements hold:

(A1) Let $j \geq k$. Then there exists a unique $s \in \mathcal{S}$ such that

$$
\operatorname{Pr}\left[S=s \mid V_{i_{1}}=v_{i_{1}}, \ldots, V_{i_{j}}=v_{i_{j}}\right]=1
$$

for any $\left\{i_{1}, \ldots, i_{j}\right\} \subseteq\{1, \ldots, n\}$ and any $\left(v_{i_{1}}, \ldots, v_{i_{j}}\right)$ with $\operatorname{Pr}\left[V_{i_{1}}=v_{i_{1}}, \ldots, V_{i_{j}}=\right.$ $\left.v_{i_{j}}\right]>0$. 
(A2) Let $j<k$. Then for each $s \in \mathcal{S}$,

$$
\operatorname{Pr}\left[S=s \mid V_{i_{1}}=v_{i_{1}}, \ldots, V_{i_{j}}=v_{i_{j}}\right]=\operatorname{Pr}[S=s]
$$

for any $\left\{i_{1}, \ldots, i_{j}\right\} \subseteq\{1, \ldots, n\}$ and any $\left(v_{i_{1}}, \ldots, v_{i_{j}}\right)$ with $\operatorname{Pr}\left[V_{i_{1}}=v_{i_{1}}, \ldots, V_{i_{j}}=\right.$ $\left.v_{i_{j}}\right]>0$.

Now we consider $k-1$ malicious participants who aim to cheat an honest one by opening forged shares and causing the honest participant to reconstruct the wrong secret.

Definition 3. For $A=\left\{i_{1}, \cdots, i_{k}\right\}$ and $v_{i_{1}} \in \mathcal{V}_{i_{1}}, \ldots, v_{i_{k}} \in \mathcal{V}_{i_{k}}$, define

$\operatorname{Sec}_{I}\left(v_{i_{1}}, \ldots, v_{i_{k}}\right)= \begin{cases}s & \text { if } \exists s \in \mathcal{S} \text { s.t. } \operatorname{Pr}\left[S=s \mid V_{i_{1}}=v_{i_{1}}, \cdots, V_{i_{k}}=v_{i_{k}}\right]=1, \\ \perp & \text { otherwise. }\end{cases}$

That is, $\operatorname{Sec}_{I}\left(v_{i_{1}}, \ldots, v_{i_{k}}\right)$ denotes the secret reconstructed from the $k$ possible shares $\left(v_{i_{1}}, \ldots, v_{i_{k}}\right)$ associated with $\left(P_{i_{1}}, \ldots, P_{i_{k}}\right)$, respectively. The symbol $\perp$ is used to indicate when no secret can be reconstructed from the $k$ shares. We will often aggregate the first $k-1$ arguments of $\operatorname{Sec}_{I}$ into a vector, by defining $\mathbf{b}=\left(v_{i_{1}}, \ldots, v_{i_{k-1}}\right)$ and $\operatorname{Sec}_{I}\left(\mathbf{b}, v_{i_{k}}\right)=\operatorname{Sec}_{I}\left(v_{i_{1}}, \ldots, v_{i_{k}}\right)$.

Definition 4. Suppose that $k-1$ cheaters $P_{i_{1}}, \ldots, P_{i_{k-1}}$ possesses the list of shares $\mathbf{b}=\left(v_{i_{1}}, \ldots, v_{i_{k-1}}\right)$. Let $\mathbf{b}^{\prime}=\left(v_{i_{1}}^{\prime}, \ldots, v_{i_{k-1}}^{\prime}\right) \neq \mathbf{b}$ be a list of $k-1$ forged shares. Then we say that $P_{i_{k}}$ is cheated by $\mathbf{b}^{\prime}$ if

$$
\operatorname{Sec}_{I}\left(\mathbf{b}^{\prime}, v_{i_{k}}\right) \notin\left\{\operatorname{Sec}_{I}\left(\mathbf{b}, v_{i_{k}}\right), \perp\right\},
$$

where $v_{i_{k}}$ denotes the share of $P_{i_{k}}$.

To define a secure secret sharing scheme clearly, we consider the following game.

1. $k-1$ cheaters and the target participant are fixed. That is, we fix $i_{1}, \ldots, i_{k-1}$ and $i_{k}$.

2. The dealer picks $s \in \mathcal{S}$ according to distribution $S$, and uses $D$ to compute shares $v_{1}, \ldots, v_{n}$ for the $n$ participants. $v_{i}$ is given to $P_{i}$ for $i \in\{1, \ldots, n\}$.

3. Let $\mathbf{b}=\left(v_{i_{1}}, \ldots, v_{i_{k-1}}\right)$. The cheaters jointly use a probabilistic algorithm $A$ to compute forged shares $\mathbf{b}^{\prime}=\left(v_{i_{1}}^{\prime}, \ldots, v_{i_{k-1}}^{\prime}\right)$ from $\mathbf{b}$.

4. The cheaters open the forged shares $\mathbf{b}^{\prime}$. If $P_{i_{k}}$ is cheated by $\mathbf{b}^{\prime}$ (as defined above), then we say that the cheaters win the cheating game.

Definition 5. We say that a $(k, n)$ threshold secret sharing scheme $D$ is a $(k, n, \delta)$ secure secret sharing scheme if

$$
\operatorname{Pr}(\text { cheaters win }) \leq \delta
$$

for any $k-1$ cheaters $P_{i_{1}}, \ldots, P_{i_{k-1}}$, any target $P_{i_{k}}$ and any cheating strategy. 
Ogata et al. derived a lower bound on $\left|\mathcal{V}_{i}\right|$ of $(k, n, \delta)$ secure secret sharing schemes as follows [4].

Proposition 1. [4] In a $(k, n, \delta)$ secure secret sharing scheme,

$$
\left|\mathcal{V}_{i}\right| \geq \frac{|\mathcal{S}|-1}{\delta}+1
$$

for any $i$.

We say that a $(k, n, \delta)$ secure secret sharing scheme is optimal if the above equality is satisfied for all $i$.

\section{Equivalence}

In this section, we show an equivalence between $(t, \delta)$-secure (1-round, $n$-channel) message transmission schemes and $(t+1, n, \delta)$ secure secret sharing schemes.

\subsection{From Secret Sharing to Message Transmission}

Theorem 1. Suppose that $n \geq 2 t+1$. If there exists a $(t+1, n, \delta)$ secure secret sharing scheme $D$ for $S$, then there exists a $(t, \epsilon)$-secure (1-round, n-channel) message transmission scheme (Enc, Dec) for the same $S$ such that

$$
\epsilon=\left(\left(\begin{array}{c}
n \\
t+1
\end{array}\right)-\left(\begin{array}{c}
n-t \\
t+1
\end{array}\right)\right) \delta
$$

Further it holds that $\mathcal{X}_{i}=\mathcal{V}_{i}$ for $1 \leq i \leq n$.

Proof. We construct (Enc, Dec) from $D$ as follows. Enc is the same as $D$. That is, on input $s \in \mathcal{S}$, Enc runs $D(s)$ to generate $\left(x_{1}, \cdots, x_{n}\right)=\left(v_{1}, \cdots, v_{n}\right)$.

Our Dec is constructed as follows. On input $\left(\hat{x}_{1}, \cdots, \hat{x}_{n}\right)$, Dec computes $\operatorname{Sec}_{I}\left(\hat{x}_{i_{1}}, \cdots, \hat{x}_{i_{t+1}}\right)$ for all $I=\left(i_{1}, \cdots, i_{t+1}\right)$, where $I$ is a subset of $\{1, \cdots, n\}$. If there exists some $\hat{s} \in \mathcal{S}$ such that

$$
\operatorname{Sec}_{I}\left(\hat{x}_{i_{1}}, \cdots, \hat{x}_{i_{t+1}}\right)=\hat{s} \text { or } \perp
$$

for all $I=\left(i_{1}, \cdots, i_{t+1}\right)$, then Dec outputs $\hat{s}$. Otherwise, Dec outputs failure.

We prove that the conditions of Def. 1 are satisfied. The privacy condition holds from (A1) of Def. 2.

Next note that

$$
n-t \geq(2 t+1)-t=t+1 \text {. }
$$

Therefore, the trivial reliability holds from (A2) of Def. 2. We next show the general reliability. From eq.(6), there exists a $J=\left\{j_{1}, \cdots, j_{t+1}\right\}$ such that $\hat{x}_{j_{1}}=$ $x_{j_{1}}, \cdots, \hat{x}_{j_{t+1}}=x_{j_{t+1}}$. For this $J$, it holds that

$$
\operatorname{Sec}_{J}\left(\hat{x}_{j_{1}}, \cdots, \hat{x}_{j_{t+1}}\right)=s
$$


from (A2) of Def. 2, where $s$ is the original secret. Therefore, Dec outputs failure if there exists some $I=\left(i_{1}, \cdots, i_{t+1}\right) \neq J$ such that

$$
\operatorname{Sec}_{I}\left(\hat{x}_{i_{1}}, \cdots, \hat{x}_{i_{t+1}}\right)=s^{\prime} \in \mathcal{S}
$$

with $s^{\prime} \neq s$. This means that if Dec does not output failure, then there is no such $I$. Hence Dec outputs $\hat{s}=s$.

Finally we show

$$
\operatorname{Pr}(\text { Dec outputs failure })<\left(\left(\begin{array}{c}
n \\
t+1
\end{array}\right)-\left(\begin{array}{c}
n-t \\
t+1
\end{array}\right)\right) \delta .
$$

For simplicity, suppose that an adversary $\mathbf{A}$ corrupts channels $1, \cdots, t$ and forges $\mathbf{b}^{\prime}=\left(x_{1}^{\prime}, \cdots, x_{t}^{\prime}\right)$. Then the number of subsets $I$ of size $t+1$ such that $I \cap$ $\{1, \cdots, t\} \neq \emptyset$ is given by $\left(\begin{array}{c}n \\ t+1\end{array}\right)-\left(\begin{array}{c}n-t \\ t+1\end{array}\right)$.

\subsection{From Message Transmission to Secret Sharing}

Suppose that there exists a $(t, \delta)$-secure (1-round, $n$-channel) message transmission scheme such that $n=2 t+1$. Then $n-t=(2 t+1)-t=t+1$. Hence from the trivial reliability condition, we can define a function $F_{I}$ such that

$$
F_{I}\left(\hat{x}_{i_{1}}, \cdots, \hat{x}_{i_{t+1}}\right)=s_{I} \text { or } \perp
$$

for each $(t+1)$-subset $I=\left(i_{1}, \cdots, i_{t+1}\right) \subset\{1, \cdots, n\}$, where $s_{I} \in \mathcal{S}$. We say that a $(t, \delta)$-secure (1-round, $n$-channel) message transmission scheme with $n=2 t+1$ is canonical if

$\operatorname{Dec}\left(\hat{x}_{1}, \cdots, \hat{x}_{n}\right)=\left\{\begin{array}{cl}\hat{s} & \text { if } F_{I}\left(\hat{x}_{i_{1}}, \cdots, \hat{x}_{i_{t+1}}\right)=\hat{s} \text { or } \perp \text { for each }(t+1) \text {-subset } I \\ \text { failure } & \text { otherwise }\end{array}\right.$

Theorem 2. If there exists a canonical $(t, \delta)$-secure (1-round, $n$-channel) message transmission scheme (Enc, Dec) with $n=2 t+1$ for $S$, then there exists a $(t+1, n, \delta)$ secure secret sharing scheme $D$ for the same $S$. Further it holds that $\mathcal{X}_{i}=\mathcal{V}_{i}$ for $1 \leq i \leq n$.

Proof. We construct $D$ from (Enc, Dec) as $D=$ Enc. That is, on input $s \in \mathcal{S}$, $D$ runs $\operatorname{Enc}(s)$ to generate $\left(v_{1}, \cdots, v_{n}\right)=\left(x_{1}, \cdots, x_{n}\right)$.

We prove that the conditions of Def. 2 are satisfied. (A1) holds from the privacy condition of Def. 1. (A2) holds from the trivial reliability since $n-t=$ $2 t+1-t=t+1$.

We finally show eq.(4). Suppose that eq.(4) does not hold in the $(t+1, n, \delta)$ secure secret sharing scheme. Then there exist some $\left\{i_{1}, \cdots, i_{t}\right\}$, a target $i_{t+1}$ and some cheating strategy such that

$$
\operatorname{Sec}_{I}\left(\mathbf{b}^{\prime}, v_{i_{k}}\right) \notin\left\{\operatorname{Sec}_{I}\left(\mathbf{b}, v_{i_{k}}\right), \perp\right\}
$$

with probability more than $\delta$. 
For simplicity, suppose that $\left\{i_{1}, \cdots, i_{t}\right\}=\{1,2, \cdots, t\}$ and $i_{t+1}=t+1$. Now in the attack game of the $(t, \delta)$-secure (1-round, $n$-channel) message transmission scheme, consider an adversary $\mathbf{A}$ which chooses the corresponding $t$ channels $\{1,2, \cdots, t\}$ and forges $x_{1}, \cdots, x_{t}$ to $x_{1}^{\prime}, \cdots, x_{t}^{\prime}$ according to the cheating strategy above. Then

$$
\operatorname{Sec}_{I}\left(x_{1}^{\prime}, \cdots, x_{t}^{\prime}, x_{t+1}\right)=s^{\prime}
$$

with probability more than $\delta$ for some $s^{\prime} \neq s$, where $I=\{1, \cdots, t, t+1\}$. On the other hand, we have

$$
\operatorname{Sec}_{J}\left(x_{t+1}, \cdots, x_{2 t+1}\right)=s
$$

for $J=\{t+1, \cdots, 2 t+1\}$. In this case, Dec outputs failure from our definition of canonical. Hence

$$
\operatorname{Pr}(\text { Dec outputs failure })>\delta \text {. }
$$

However, this is against eq.(2). Therefore, eq.(4) must hold.

\subsection{Discussion}

We show that canonical is a natural property that $(t, \delta)$-secure (1-round, $n$ channel) message transmission schemes with $n=2 t+1$ should satisfy. First from the proof of Theorem 1, we have the following corollary.

Corollary 1. In Theorem 1 , if $n=2 t+1$, then the message transmission scheme is canonical.

Next suppose that there exists a $(t, \delta)$-secure (1-round, $n$-channel) message transmission scheme with $n=2 t+1$. Remember that the sender sends a ciphertext $\left(x_{1}, \cdots, x_{2 t+1}\right)$ for a secret $s$, and the receiver receives $\hat{X}=\left(\hat{x}_{1}, \cdots, \hat{x}_{n}\right)$. For a $(t+1)$-subset $I=\left(i_{1}, \cdots, i_{t+1}\right) \subset\{1, \cdots, n\}$, define

$$
G(I, \hat{X})=F_{I}\left(\hat{x}_{i_{1}}, \cdots, \hat{x}_{i_{t+1}}\right) .
$$

(See eq. (7) for $F_{I}$.)

Definition 6. We say that a $(t+1)$-subset I is an acceptable (sub) set for $\hat{X}$ if $G(I, \hat{X}) \neq \perp$.

In a canonical scheme, it is easy to see that Dec outputs failure if and only if there exist two acceptable $(t+1)$-subsets $I$ and $J$ such that $G(I, \hat{X}) \neq$ $G(J, \hat{X})$. We will show that this is a natural property that $(t, \delta)$-secure (1-round, $n$-channel) message transmission schemes with $n=2 t+1$ should satisfy.

Consider an adversary $\mathbf{A}$ who corrupts channels $1, \cdots, t$, and replaces $x_{i}$ to a random $x_{i}^{\prime}$ for $1 \leq i \leq t$.

1. We first show that

- there are only two acceptable sets $I$ and $J$, and $G(I, \hat{X}) \neq G(J, \hat{X})$ 
with nonzero probability. In this case, the receiver cannot see if $G(I, \hat{X})=$ $s$ or $G(J, \hat{X})=s$. Hence he must output failure to satisfy the general reliability condition.

The proof is as follows. From the trivial reliability, it holds that

$$
G(I, \hat{X})=s
$$

for $I=\{t+1, \cdots, 2 t+1\}$. Further there exists another acceptable set $J \neq I$ such that $G(I, \hat{X}) \neq G(J, \hat{X})$ with nonzero probability. Because otherwise we have a perfectly $t$-secure (1-round, $n$-channel) message transmission scheme with $n=2 t+1$, which is a contradiction.

Finally, there exist no other acceptable sets with high probability because $x_{i}^{\prime}$ is chosen randomly for $1 \leq i \leq t$.

2. Next we show that there exists a case such that the majority vote does not work. That is, we show that there exist acceptable sets $I$ and $J_{1}, \cdots, J_{\left(\begin{array}{c}2 t \\ t+1\end{array}\right)}$ such that

$$
\begin{aligned}
& -G(I, \hat{X})=s \text { and } \\
& -G\left(J_{1}, X\right)=\cdots, G\left(J_{\left(\begin{array}{c}
2 t \\
t+1
\end{array}\right)}, \hat{X}\right)=s^{\prime} \neq s
\end{aligned}
$$

with nonzero probability. In this case, the receiver must output failure too to satisfy the general reliability condition.

The proof is as follows. From the privacy condition, we have no information on $s$ from $\left(x_{t+1}, \cdots, x_{2 t}\right)$. Therefore for $s^{\prime} \neq s$, it holds that

$$
\operatorname{Pr}\left[S=s^{\prime}, X_{t+1}=x_{t+1}, \cdots, X_{2 t}=x_{2 t}\right]>0 .
$$

Hence there exist some $b_{1}, \cdots, b_{t}, c_{2 t+1}$ such that

$\operatorname{Pr}\left[S=s^{\prime}, X_{1}=b_{1}, \cdots, X_{t}=b_{t}, X_{t+1}=x_{t+1}, \cdots, X_{2 t}=x_{2 t}, X_{2 t+1}=c_{2 t+1}\right]>0$.

Further it holds that $x_{i}^{\prime}=b_{i}$ for $1 \leq i \leq t$ with nonzero probability because the adversary $\mathbf{A}$ chooses $x_{i}^{\prime}$ randomly. In this case, we have

$$
\hat{x}_{1}=b_{1}, \cdots, \hat{x}_{t}=b_{t}, \hat{x}_{t+1}=x_{t+1}, \cdots, \hat{x}_{2 t}=x_{2 t}, \hat{x}_{2 t+1}=x_{2 t+1} .
$$

Therefore from eq.(11), for any $(t+1)$-subset $J \subset\{1, \cdots, 2 t\}$, we obtain that

$$
G(J, \hat{X})=s^{\prime}
$$

The number of such $J$ is $\left(\begin{array}{c}2 t \\ t+1\end{array}\right)$. Finally, it is clear that $G(I, \hat{X})=s$ for $I=\{t+1, \cdots, 2 t+1\}$.

So the scheme must be canonical in the above two cases. Hence we consider that canonical is a natural property for $n=2 t+1$.

\section{Lower Bound}

In this section, we derive a lower bound on $\left|\mathcal{X}_{i}\right|$ of $(t, \delta)$-secure (1-round, $n$ channel) message transmission schemes with $n=2 t+1$ by using our equivalence. Indeed, we obtain the following bound immediately from Proposition 1 and Theorem 2. 
Corollary 2. In a canonical $(t, \delta)$-secure (1-round, n-channel) message transmission scheme with $n=2 t+1$, it holds that

$$
\left|\mathcal{X}_{i}\right| \geq \frac{|\mathcal{S}|-1}{\delta}+1
$$

for any $i$.

\section{Near Optimum Almost Secure MT Scheme}

Ogata et al. showed a construction of optimum $(k, n, \delta)$ secure secret sharing schemes by using "difference sets" [4].

\subsection{Optimum Robust Secret Sharing Scheme}

Definition 7. [3, p.397] $A$ planar difference set modulo $N=\ell(\ell-1)+1$ is a set $B=\left\{d_{0}, d_{1}, \ldots, d_{\ell-1}\right\} \subseteq \mathbb{Z}_{N}$ with the property that the $\ell(\ell-1)$ differences $d_{i}-d_{j}\left(d_{i} \neq d_{j}\right)$, when reduced modulo $N$, are exactly the numbers $1,2, \ldots, N-1$ in some order.

For example, $\left\{d_{0}=0, d_{1}=1, d_{2}=3\right\}$ is a planar difference set modulo 7 with $\ell=3$. Indeed, the differences modulo 7 are

$$
1-0=1,3-0=3,3-1=2,0-1=6,0-3=4,1-3=5 .
$$

Proposition 2. [3, p.398, Theorem 22] Let $\Pi$ be a projective plane $P G(2, q)$. $A$ point in $\Pi$ can be represented as $\left(\beta_{1}, \beta_{2}, \beta_{3}\right) \in\left(\mathbb{F}_{q}\right)^{3}$, or $\alpha^{i} \in \mathbb{F}_{q^{3}}$ for some $i$, where $\alpha$ is a generator of $\mathbb{F}_{q^{3}}$. If $\ell=q+1$ points $\alpha^{d_{0}}, \ldots, \alpha^{d_{\ell-1}}$ are the points on a line in $\Pi$, then $\left\{d_{0}, \ldots, d_{\ell-1}\right\}$ is a planar difference set modulo $q^{2}+q+1$.

Let $\left\{d_{0}, \ldots, d_{q}\right\}$ be a planar difference set modulo $p=q^{2}+q+1$. Then a $(k, n, \delta)$ secure secret sharing scheme is obtained by applying Shamir's $(k, n)$ threshold secret sharing scheme to $\mathcal{S}=\left\{d_{0}, \ldots, d_{q}\right\}$ over $G F(p)$, where the secret $s$ is uniformly distributed over $\mathcal{S}$ and $\delta=1 /(q+1)$. In the reconstructoin phase, an honest participant outputs a reconstructed secret $s^{\prime}$ if $s^{\prime} \in \mathcal{S}$, and $\perp$ otherwise.

Proposition 3. [4, Corollary 4.5] Let $q$ be a prime power that makes $q^{2}+q+1$ a prime. Then, there exists a $(k, n, \delta)$ secure secret sharing scheme for a uniform distribution over $\mathcal{S}$ which meets the bound (5) such that $|\mathcal{S}|=q+1, \delta=1 /(q+1)$ and $n<q^{2}+q+1$.

From Proposition 1, this construction is optimum. 


\subsection{Near Optimum Almost Secure MT Scheme}

From the above proposition, Theorem 1 and Corollary 1, we can obtain the following construction of $(t, \epsilon)$-secure (1-round, $n$-channel) message transmission schemes.

Corollary 3. Let $q$ be a prime power that makes $q^{2}+q+1$ a prime. Then, there exists a $(t, \epsilon)$-secure (1-round, $n$-channel) message transmission scheme with $n \geq 2 t+1$ for a uniform distribution over $\mathcal{S}$ such that $|\mathcal{S}|=q+1, \delta=$ $1 /(q+1), 2 t+1 \leq n<q^{2}+q+1$ and

$$
\left|\mathcal{X}_{i}\right|=\frac{|\mathcal{S}|-1}{\delta}+1,
$$

where

$$
\epsilon=\left(\left(\begin{array}{c}
n \\
t+1
\end{array}\right)-\left(\begin{array}{c}
n-t \\
t+1
\end{array}\right)\right) \delta .
$$

Further if $n=2 t+1$, the message transmission scheme is canonical.

Our $(t, \epsilon)$-secure (1-round, $n$-channel) message transmission scheme is described as follows. Let $\left\{d_{0}, \ldots, d_{q}\right\}$ be a planar difference set modulo $p=q^{2}+$ $q+1$. We assume that a message $s$ is uniformly distributed over $\{0,1, \cdots, q\}$.

1. For a message $s \in\{0,1, \ldots, q\}$, let $y=d_{s}$. The sender applies Shamir's $(t+1, n)$-threshold secret sharing scheme to the secret $y=d_{s}$ over $G F(p)$ to obtain the shares $\left(v_{1}, \cdots, v_{n}\right)$. She then sends $x_{i}=v_{i}$ to the receiver through the $i$ th channel for $i=1, \cdots, n$.

2. Suppose that the receiver received $\left(\hat{x}_{1}, \cdots, \hat{x}_{n}\right)$. He first reconstructs the secret $y_{I}$ by applying Lagrange formula to $\left(\hat{x}_{i_{1}}, \cdots, \hat{x}_{i_{t+1}}\right)$ for each subset $I=\left(i_{1}, \cdots, i_{t+1}\right)$ of $\{1, \cdots, n\}$. If there exists some $\hat{s} \in\{0,1, \ldots, q\}$ such that for all subset $I$,

$$
y_{I}=d_{\hat{s}} \text { or } y_{I} \notin\left\{d_{0}, \ldots, d_{q}\right\},
$$

then he outputs $\hat{s}$. Otherwise the receiver outputs failure.

\subsection{Generalization}

Ogata et al. also showed another construction of optimum $(k, n, \delta)$ secure secret sharing schemes by using general "difference sets" [4].

Proposition 4. [4, Corollary 4.5] For a positive integer $u$ such that $4 u-1$ is a prime power, there exists a $(k, n, \delta)$ secure secret sharing scheme which meets the equality of our bound (5), such that $|\mathcal{S}|=2 u-1, \delta=(u-1) /(2 u-1), n<4 u-1$.

From the above proposition, Theorem 1 and Corollary 1, we can obtain another construction of $(t, \epsilon)$-secure (1-round, $n$-channel) message transmission schemes as follows. 
Corollary 4. [4, Corollary 4.5] For a positive integer u such that $4 u-1$ is a prime power, there exists $(t, \epsilon)$-secure (1-round, $n$-channel) message transmission scheme with $n \geq 2 t+1$ for a uniform distribution over $\mathcal{S}$ such that $|\mathcal{S}|=$ $2 u-1, \delta=(u-1) /(2 u-1), n<4 u-1$ and

$$
\left|\mathcal{X}_{i}\right|=\frac{|\mathcal{S}|-1}{\delta}+1
$$

where

$$
\epsilon=\left(\left(\begin{array}{c}
n \\
t+1
\end{array}\right)-\left(\begin{array}{c}
n-t \\
t+1
\end{array}\right)\right) \delta
$$

Further if $n=2 t+1$, the message transmission scheme is canonical.

In these constructions, there is a gap of $\left.\log _{2}\left(\begin{array}{c}n \\ t+1\end{array}\right)-\left(\begin{array}{c}n-t \\ t+1\end{array}\right)\right)$ bits from our lower bound of Corollary 2. This gap is, however, small enough for small $t$.

Our results imply that $n \geq 2 t+1$ is necessary and sufficient for $(t, \epsilon)$-secure (1-round, $n$-channel) message transmission schemes.

Theorem 3. $(t, \epsilon)$-secure (1-round, $n$-channel) message transmission schemes exist if and only if $n \geq 2 t+1$.

Proof. It is enough to prove that there exist no $(t, \epsilon)$-secure (1-round, $n$-channel) message transmission schemes for $n \leq 2 t$. Suppose that there exists a $(t, \epsilon)$ secure (1-round, $n$-channel) message transmission scheme with $n \leq 2 t$. Consider an adversary $\mathbf{A}$ who replaces $x_{1}, \cdots, x_{t}$ with null strings. Then the receiver receives $n-t$ messages $x_{t+1}, \cdots, x_{n}$, where $n-t \leq 2 t-t=t$. Then from the privacy condition, the receiver obtains no information on $s$. On the other hand, from the trivial reliability condition, he must output $s$. This is a contradiction.

\section{Conclusion}

In this paper, we first summed up a number of flaw of the previous almost secure (1-round, $n$-channel) message transmission scheme for $n=2 t+1$ which was presented at Crypto 2004. We next showed an equivalence between $(t, \delta)$-secure (1-round, $n$-channel) message transmission scheme for $n=2 t+1$ and secret sharing schemes with cheaters. By using our equivalence, we derived a lower bound on the communication complexity. Finally, we presented a near optimum scheme which meets our bound approximately. This is the first construction of provably secure $(t, \delta)$-secure (1-round, $n$-channel) message transmission schemes for $n=2 t+1$.

Our results imply that $n \geq 2 t+1$ is necessary and sufficient for $(t, \epsilon)$-secure (1-round, $n$-channel) message transmission schemes. 


\section{References}

1. S.Agarwal, R.Cramer, and R. de Haan: Asymptotically Optimal Two-Round Perfectly Secure Message Transmission CRYPTO 2006: 394-408

2. D.Dolev, C.Dwork, O.Waarts, M.Yung: Perfectly Secure Message Transmission. J. ACM 40(1): 17-47 (1993)

3. F.J. MacWilliams and N.J.A. Sloanem The Theory of Error-correcting Codes, North-Holland, 1981

4. W.Ogata, K.Kurosawa, D.Stinson: Optimum Secret Sharing Scheme Secure against Cheating. SIAM J. Discrete Math. 20(1): 79-95 (2006)

5. H.Md.Sayeed and H.Abu-Amara: Efficient Perfectly Secure Message Transmission in Synchronous Networks. Inf. Comput. 126(1): 53-61 (1996)

6. K. Srinathan, A.Narayanan, C. Pandu Rangan: Optimal Perfectly Secure Message Transmission. CRYPTO 2004: 545-561

7. M. Tompa and H. Woll, How to share a secret with cheaters, Journal of Cryptology 1 (1988), 133-138. 